\title{
Large-scale influences in near-wall turbulence
}

\author{
By Nicholas Hutchins* and Ivan Marusic \\ Walter Bassett Aerodynamics Laboratory, Mechanical and Manufacturing \\ Engineering, University of Melbourne, Victoria 3010, Australia
}

Hot-wire data acquired in a high Reynolds number facility are used to illustrate the need for adequate scale separation when considering the coherent structure in wall-bounded turbulence. It is found that a large-scale motion in the log region becomes increasingly comparable in energy to the near-wall cycle as the Reynolds number increases. Through decomposition of fluctuating velocity signals, it is shown that this large-scale motion has a distinct modulating influence on the small-scale energy (akin to amplitude modulation). Reassessment of DNS data, in light of these results, shows similar trends, with the rate and intensity of production due to the near-wall cycle subject to a modulating influence from the largest-scale motions.

Keywords: superstructures; coherent structures; amplitude modulation

\section{Introduction}

Over the past several decades, there has been an emergent understanding that wall-bounded turbulence is composed of certain recurrent and quantifiable features, collectively termed 'coherent structures'. The most widely recognized of these is the near-wall cycle of quasi-streamwise vortices, originally visualized as streakiness in the velocity field of the viscous sublayer (e.g. Kline et al. 1967). The dominant spanwise spacing and length-scale of these near-wall streaks are found to scale on viscous wall units (a function of friction velocity $U_{\tau}$ and the kinematic viscosity $\nu$ ). The importance of the near-wall cycle is undeniable. These features make up a well-defined stress-producing cycle that leads to the large peak in near-wall turbulence production and thus they play a key role in creating and sustaining this turbulent flow. However, until quite recently, the vast majority of studies of coherent motions have been conducted at low Reynolds numbers, where the separation of scales is by definition limited. Reynolds number, often defined as the ratio of inertial to viscous forces, can also be viewed as a measure of separation between the largest and the smallest scales (the ratio of inertial to viscous scales).

More recent advances in particle image velocimetry (PIV) and large numerical domain direct numerical simulations (DNS) have provided new opportunities to study the structure of wall-bounded turbulence at extended Reynolds number

\footnotetext{
* Author for correspondence (nhu@unimelb.edu.au).
}

One contribution of 14 to a Theme Issue 'Scaling and structure in high Reynolds number wallbounded flows'. 
ranges (e.g. Adrian et al. 2000b; del Álamo et al. 2004). The ability of these two techniques to provide instantaneous planar and volumetric fields has enabled new insights into coherent motions, proving particularly successful in uncovering a class of much larger scale motions that dominate the logarithmic and wake regions of the boundary layer.

An assumption of universal behaviour in the near-wall region, based on viscous scaling, implies an underlying belief that the role of these large outer-scale motions remains passive at all Reynolds numbers. This paper makes use of hotwire data, acquired at moderately high Reynolds numbers, to question the basis of this assumption. By contrasting the energy content at different scales, we highlight an increasing influence of the largest outer-scaled structures with increasing Reynolds number. As well as illustrating the need for adequate scale separation (and hence Reynolds number) when assessing the true range of coherent motions, these data also appear to show that the emergent large-scale structure imposes a modulating influence on the viscous-scaled near-wall cycle. In doing so, we demonstrate how a relatively simple (single-point) high Reynolds number measurement can help us reassess and interpret more complex (volumetric and planar) lower Reynolds number DNS and PIV data.

\section{Need for high Reynolds number studies}

To explain the need for adequately high Reynolds number, we will consider the energy spectra of streamwise velocity fluctuations for a turbulent boundary layer at $R e_{\tau}=7300$. Throughout this paper, the axis system, $x, y$ and $z$, refer to the streamwise, spanwise and wall-normal directions, with $u, v$ and $w$ describing the respective fluctuating velocity components. $k_{x}$ is the streamwise wavenumber and $\phi_{u u}$ is the spectral density of the streamwise velocity fluctuations. $R e_{\tau}\left(=\delta U_{\tau} / \nu\right)$ is the Reynolds number based on the boundary-layer thickness $\delta$ and friction velocity $U_{\tau}$. Capitalized velocities (e.g. $U$ ) and overbars indicate time-averaged values and the superscript ' + ' is used to denote viscous scaling of length (e.g. $\left.z^{+}=z U_{\tau} / \nu\right)$, velocities $\left(U^{+}=U / U_{\tau}\right)$ and time $\left(t^{+}=t U_{\tau}^{2} / \nu\right)$.

Figure 1 gives an overview of pre-multiplied streamwise energy spectra, $k_{x} \phi_{u u}$, across the full height of a turbulent boundary layer, together with the corresponding mean velocity and turbulence intensity profiles. These data are from hot-wire measurements conducted in the high Reynolds number boundarylayer facility at the University of Melbourne with wire length $l^{+} \approx 22$, sample interval $T^{+} \approx 0.4$ and sample length in the range 5000-14 000 boundary-layer thicknesses. (Full details of the facility are given by Nickels et al. 2005.) All data are presented in terms of streamwise length-scale $\lambda_{x}\left(=2 \pi / k_{x}\right)$. It is noted that this representation is simply a reflected mirror image of a conventional $k_{x} \phi_{u u}^{+}$ versus $\log \left(k_{x} \delta\right)$ plot, and that equal area under the curve still represents equal contribution to energy.

Considering first the near-wall region, figure $1 a$ shows the spectrum at $z^{+}=15$, a height that corresponds to the near-wall peak in turbulence production and also the peak in the broadband streamwise intensity (see closed symbols in figure $1 d$ ). At this wall-normal position, a clear peak in the pre-multiplied spectra occurs at a length-scale close to $\lambda_{x}^{+} \approx 1000$. This would indicate that the most energetic $u$ fluctuations are principally of this length (within the usual limits of sinusoidal 
(a)

$\lambda_{x}^{+}$

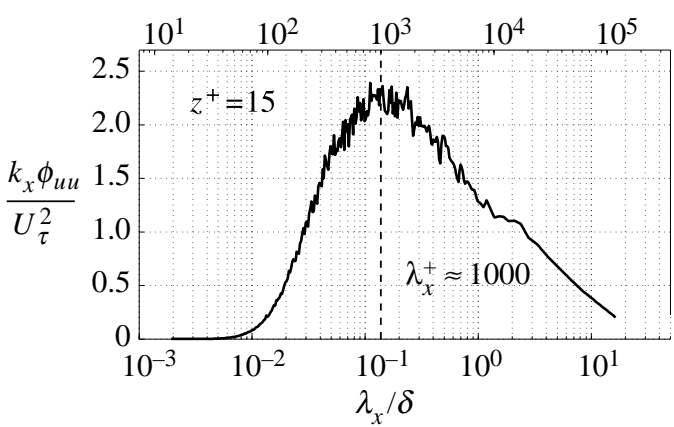

(b)

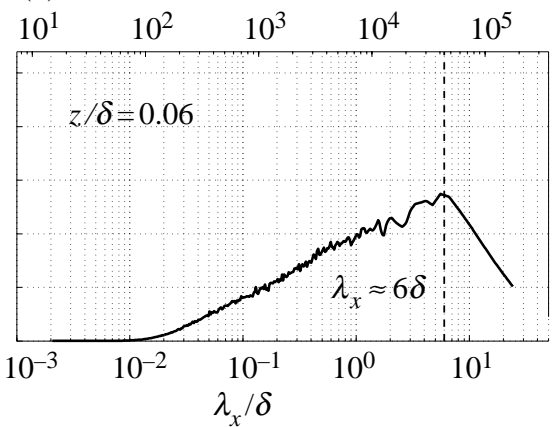

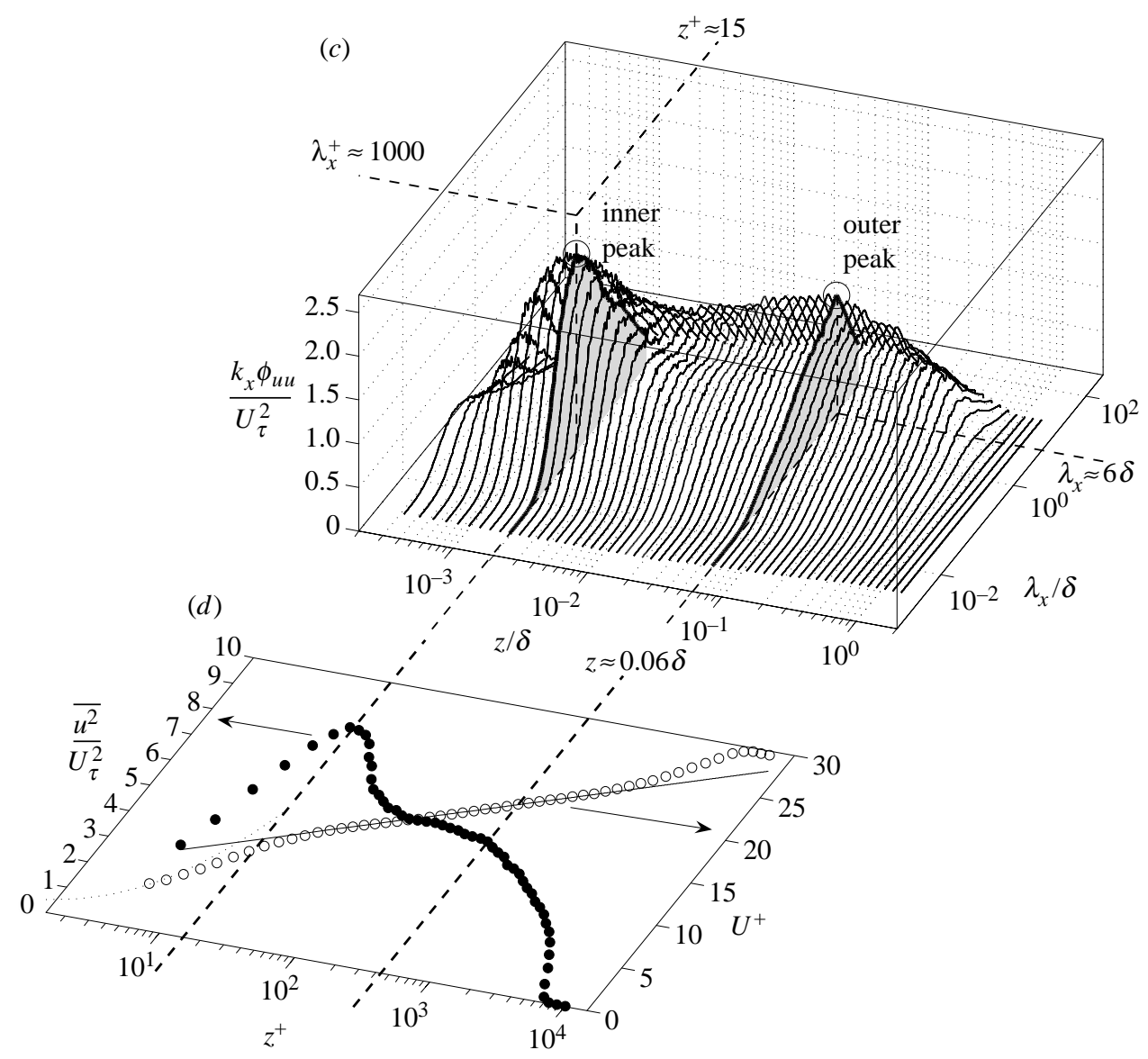

Figure 1. Pre-multiplied energy spectra of streamwise velocity fluctuation $k_{x} \phi_{u u} / U_{\tau}^{2}$ for a turbulent boundary layer at $R e_{\tau} \approx 7300$. (a) In the viscous buffer region at $z^{+} \approx 15,(b)$ in the logarithmic region at $z / \delta \approx 0.06,(c)$ as a three-dimensional surface plot for all wall-normal locations and $(d)$ corresponding (open small circle) mean velocity and (filled circle) broadband turbulence intensity profiles where the dotted line shows linear behaviour in the viscous sublayer $U^{+}=z^{+}$and the solid line shows $U^{+}=(1 / \kappa) \ln \left(z^{+}\right)+A$ (where $\kappa=0.41$ and $A=5.0$ ). For all plots, the dashed lines denote locations of inner and outer spectral peaks. 
decomposition). This peak is the energetic signature due to the viscous-scaled near-wall structure of elongated high- and low-speed regions (Kline et al. 1967). There are several review papers that deal with these streaks and associated vortical structure (Robinson 1991; Panton 2001) and it is not the intention to revisit these results here. For now, it is sufficient to note that there is a near-wall energy peak or site associated with these features, and that this peak is approximately fixed in viscous coordinates at $z^{+} \approx 15$ and $\lambda_{x}^{+} \approx 1000$. We will refer to this peak as the 'inner site' in accordance with Hutchins \& Marusic (in press).

Figure $1 b$ shows the streamwise pre-multiplied spectra at $z / \delta=0.06$, a height corresponding roughly to the midpoint of the logarithmic region (we assume the logarithmic portion of the velocity profile to exist between the approximate limits $z^{+}>100$ and $z / \delta<0.15$, see open symbols in figure $1 d$ ). At this height, a clear peak in the pre-multiplied spectra occurs at length-scales that are many times larger than the boundary-layer thickness $\left(\lambda_{x} \approx 6 \delta\right)$. We will refer to this peak as the 'outer site'.

Figure $1 c$ fills in the gaps between the 'inner' and the 'outer' energy sites, showing the pre-multiplied energy spectra for streamwise fluctuations at 55 logarithmically spaced stations across the boundary layer. The surface formed from these profiles highlights the bimodal composition, with two competing energetic peaks due to both the viscous-scaled near-wall cycle (inner site) and the much larger outer-scaled events in the logarithmic region (outer site).

The spectra shown in figure 1 also include the energetic contributions from a range of other motions (including $k_{x}^{-1}$ scaling of attached eddies, outer wake-type motions, etc.). However, the prominent features we wish to highlight here are the 'inner' and 'outer' sites, where the Reynolds number determines the degree of separation between these sites. At low Reynolds numbers, there will be an increasing amount of overlap between these two scales until eventually, at some critical Reynolds number, the physical dimension of the inner and outer lengthscale will be equal. This will occur when

$$
6 \delta=\frac{1000 \nu}{U_{\tau}},
$$

giving

$$
R e_{\tau_{\text {crit }}}=\left(\frac{\delta U_{\tau}}{\nu}\right)=\frac{1000}{6} \approx 167 .
$$

Interestingly, this also represents a point where the inner and the outer scales are centred at a roughly similar height from the wall (at $R e_{\tau_{\text {crit }}}$, the outer peak at $z / \delta=0.06$ would be at $z^{+}=10$ ). Thus, at values close to $R e_{\tau_{\text {crit }}}$, there is almost complete overlap between the two scales, and no chance of distinguishing inner from outer scales. In reality, this lower limit is not such a useful criterion for experimental design, since it so closely corresponds to the turbulent limit, below which the boundary layer is normally considered stable to fluctuations. Alternatively, figure $1 c, d$ indicate that the outer site is associated with the logarithmic region, which may suggest a further possible Reynolds number limit. By our previous definition, the logarithmic region will only exist when

$$
0.15 \delta>\frac{100 \nu}{U_{\tau}},
$$


giving a lower Reynolds number limit for logarithmic behaviour of the mean velocity profile,

$$
R e_{\tau_{\log }}>\frac{100}{0.15} \geq 667 .
$$

More realistically, one would typically wish to see at least a decade of separation (one order of magnitude) between the two energy sites before a clear distinction can be made between near-wall and log-region coherent structures. This requires

$$
R e_{\tau_{\mathrm{dec}}}>\frac{10000}{6} \gtrsim 1700 \text {. }
$$

This Reynolds number has added significance, since it places the centroid of the outer-scaled energy beyond the commonly prescribed limit of the buffer region (where viscous scales dominate) and into the $\log$ region (i.e. at $R e_{\tau} \approx 1700$, $\left.z / \delta=0.06 \rightarrow z^{+}=100\right)$. These limits agree well with our own experimental observations on the emergence of the outer energy site (Hutchins \& Marusic in press).

Figure 2 is included as an example of a boundary layer with a Reynolds number that falls someway below this limit $\left(R e_{\tau}=1000\right)$. The data are presented in an identical manner to figure 1 (although there are only 36 logarithmically spaced measurement stations). These hot-wire data were acquired in a low Reynolds number boundary-layer facility at the University of Minnesota with wire length $l^{+} \rightarrow 14$, sample interval $T^{+} \approx 0.14$ and total sample advection length in the range 9000-22 $000 \delta$ (for further details see Hutchins \& Marusic in press).

At $z^{+}=15$, the general form of the energy spectra is similar for the two Reynolds numbers, with a clear peak centred around $\lambda_{x}^{+} \approx 1000$ for both cases (compare figures $2 a$ and $1 a$ ). However, the log-region spectrum of figure $2 b$ has a very different form to the higher Reynolds number case. While there is a weak secondary peak at $\lambda_{x}=6 \delta$, it is largely obscured by a very strong remanent of the energy due to the near-wall structure $\left(\lambda_{x}^{+} \approx 1000\right)$. At this low Reynolds number, the two energy sites are too close, both in $z$ and $\lambda_{x}$, to see adequate distinction between scales in $k_{x} \phi_{u u}$. This is exacerbated by a reduced magnitude of the outer energy site at lower Reynolds numbers as highlighted in figure $2 c$. The outer peak, visible only as a weak ridge, is largely swamped by the near-wall viscousscaled energy and the surface looks very different from the bimodal surface that emerged in figure $1 c$. At this Reynolds number, the degree of overlap in $z$ and $\lambda_{x}$ will make it difficult to distinguish between the viscous and the outer scales in statistics (e.g. spectra, correlations, conditional averaging, etc.).

Up until very recently, much of the work on near-wall coherent motions has come from studies conducted below the limit proposed by equation (2.5) (e.g. Spalart 1988; Robinson 1991). This means not only that there has been a general tendency to study the near-wall structure in isolation from large-scale events, but also that even when investigations have sought to study the log-region structure, this has often been done at Reynolds numbers where the degree of overlap between the viscous and the outer scales may tend to mask the true asymptotic form of the log-region structure. As an example, the majority of recent studies of the log-region structure, specifically the packet literature, have tended to be PIV investigations, which by nature tend to cover a fairly limited Reynolds number range, e.g. Ganapathisubramani et al. (2003), $R e_{\tau}=1060$; 

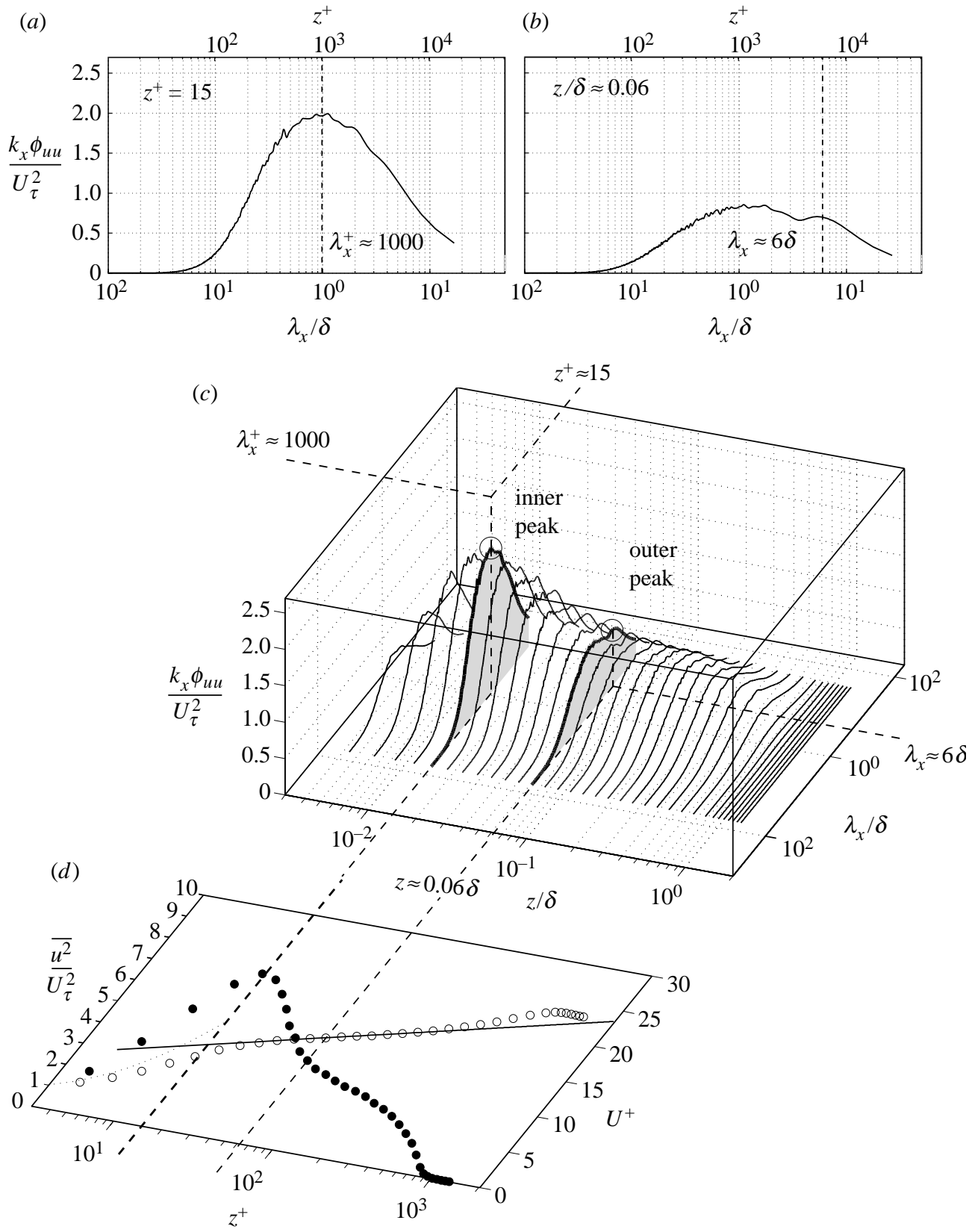

Figure 2. Pre-multiplied energy spectra of streamwise velocity fluctuation $k_{x} \phi_{u u} / U_{\tau}^{2}$ for $R e_{\tau} \approx 1000$. See the caption of figure 1 for details.

Ganapathisubramani et al. (2005), $R e_{\tau}=1060,1142$; Hutchins et al. (2005), $R e_{\tau}=690,1010,1840,2800$; Hambleton et al. (2006), $R e_{\tau}=1100$; Zhou et al. (1999), $R e_{\tau}=182$; Liu et al. (2001), $R e_{\tau}=315,1414$; Tomkins \& Adrian (2003), $R e_{\tau}=424,2226$; Adrian et al. $(2000 b), R e_{\tau}=357,833,2030$; Christensen \& Adrian (2001), $R e_{\tau}=547,1734$. 


\section{(a) Internal geometries}

Thus far, the discussion of spectra, and the Reynolds number implications, has been limited to flat-plate boundary layers. However, there is considerable evidence that the large-scale energy in pipes and channels may behave differently (certainly the external or wake component of these geometries is very different). Kim \& Adrian (1999) report streamwise length-scales of up to 12-14 pipe radii for pipe flows (based on the peak in $k_{x} \phi_{u u}$ ). Guala et al. (2006) report similar behaviour, noting also a separate dominant scale at $z \approx r / 2$ of length-scale $\lambda_{x} / r \approx 2-3$ (where $r$ is the pipe radius). We note that the spectral surfaces of the form shown in figures 1 and 2 relax to a broad maximum centred at $\lambda_{x} \approx 2-3 \delta$ in the wake region (see also figure 3 at $z \approx \delta / 2$ ). For channels, analysis of large numerical domain DNS results (in particular two-dimensional spectra) have shown that the log region $\phi_{u u}$ energy can reside in very long streamwise modes for larger $k_{y}$ bands (certainly more than 20 channel half heights; see Jiménez 1998; del Alamo \& Jiménez 2003). Abe et al. (2004) show the spanwise analogue of figures 1 and 2 for DNS of a channel flow at $R e_{\tau} \approx 640$. They show bimodal behaviour in the spectral surface of $k_{y} \phi_{u u}$, with an inner peak at $\lambda_{y}^{+} \approx 100$ and outer peak at $\lambda_{y} \approx 1.3-$ 1.6h. Kasagi et al. (2005) show similar results for $R e_{\tau}=1160$. For boundary layers, the reported spanwise scale for the outer energy site is smaller, with $\lambda_{y} \approx 0.7-0.8 \delta$ commonly reported (Marusic \& Hutchins 2005; Tomkins \& Adrian 2005). Since the outer energy site occurs at greater $\lambda_{x}$ and $\lambda_{y}$ values for internal geometries, the decade of separation between the inner and the outer scales will be achieved at lower $R e_{\tau}$ for these flows than indicated by equation (2.5).

\section{Structure in log layer}

To highlight possible motions that contribute to the 'outer' energetic site, we will briefly review some recent investigations of coherent structure in the log region of turbulent boundary layers.

PIV studies of streamwise-spanwise planes by Ganapathisubramani et al. (2003), Tomkins \& Adrian (2003) and Hambleton et al. (2006) have revealed an over-riding prevalence of stripiness in instantaneous fields of $u$ fluctuation in the log region. Long regions of streamwise momentum deficit are found, with highspeed fluid tending to fill the separation between the neighbouring motions. Analysis of swirling motions reported therein has shown that these features have an associated vortex structure which, for the Reynolds number range considered, is consistent with the hairpin packet scenario. The low-speed regions are of the order $0.3-0.5 \delta$ wide in the spanwise direction, and typically have a streamwise extent that exceeds the PIV field-of-view (usually limited to approximately $2 \delta$ ). In an effort to ascertain the true length of these features, Hutchins et al. (2004) and Hutchins \& Marusic (in press) employed spanwise rakes of 10 hot-wire sensors. The rake provides the instantaneous spanwise profile of the $u$ fluctuation, which when projected in $x$ using Taylor's hypothesis produces a view of the low- and high-speed streaks that covers a much larger streamwise domain than that attainable from PIV. These rake measurements indicated very long meandering low- and high-speed features routinely exceeding $15 \delta$ in length. Such features normally occur as alternating spanwise patterns, i.e. an elongated low-speed event will typically be flanked on either side by an elongated 
(a)

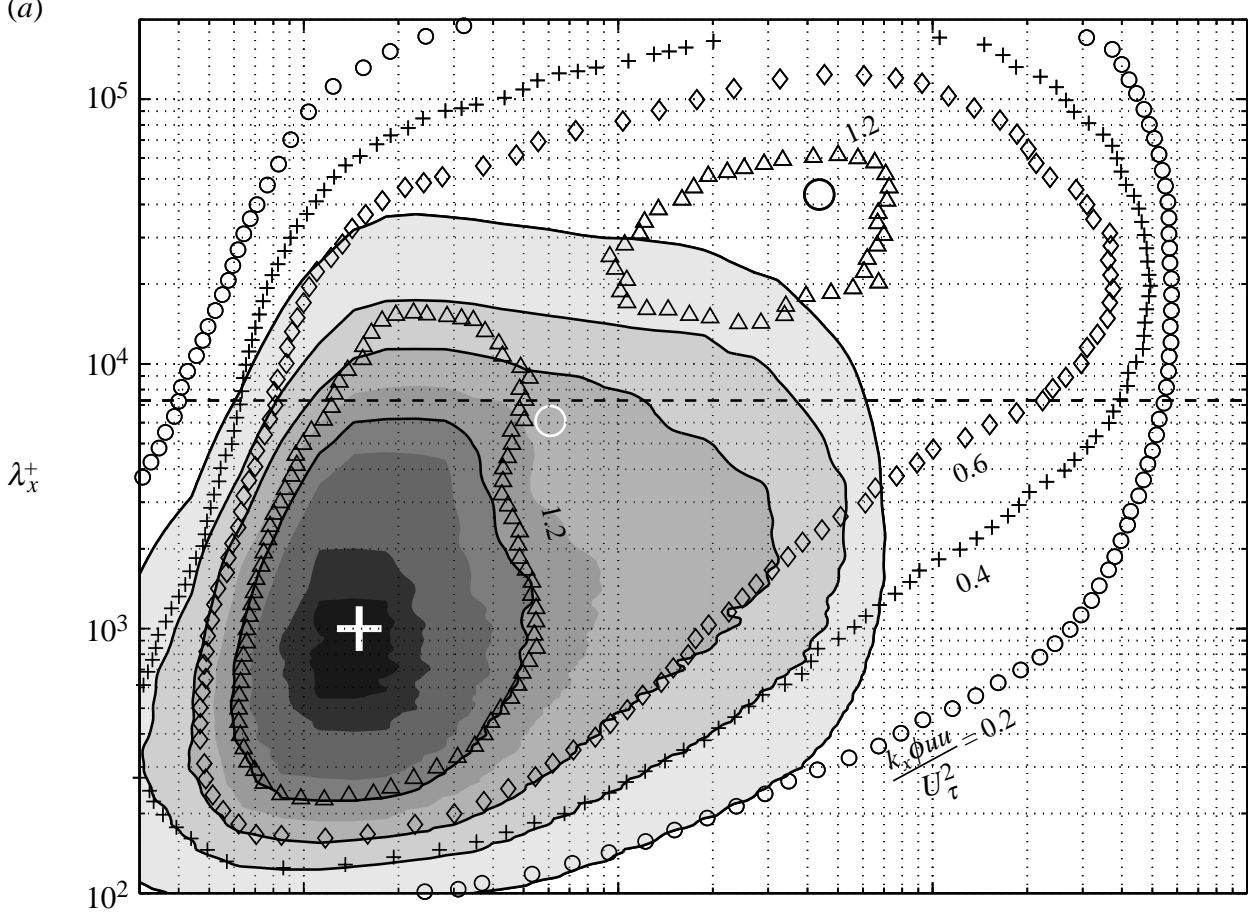

(b)

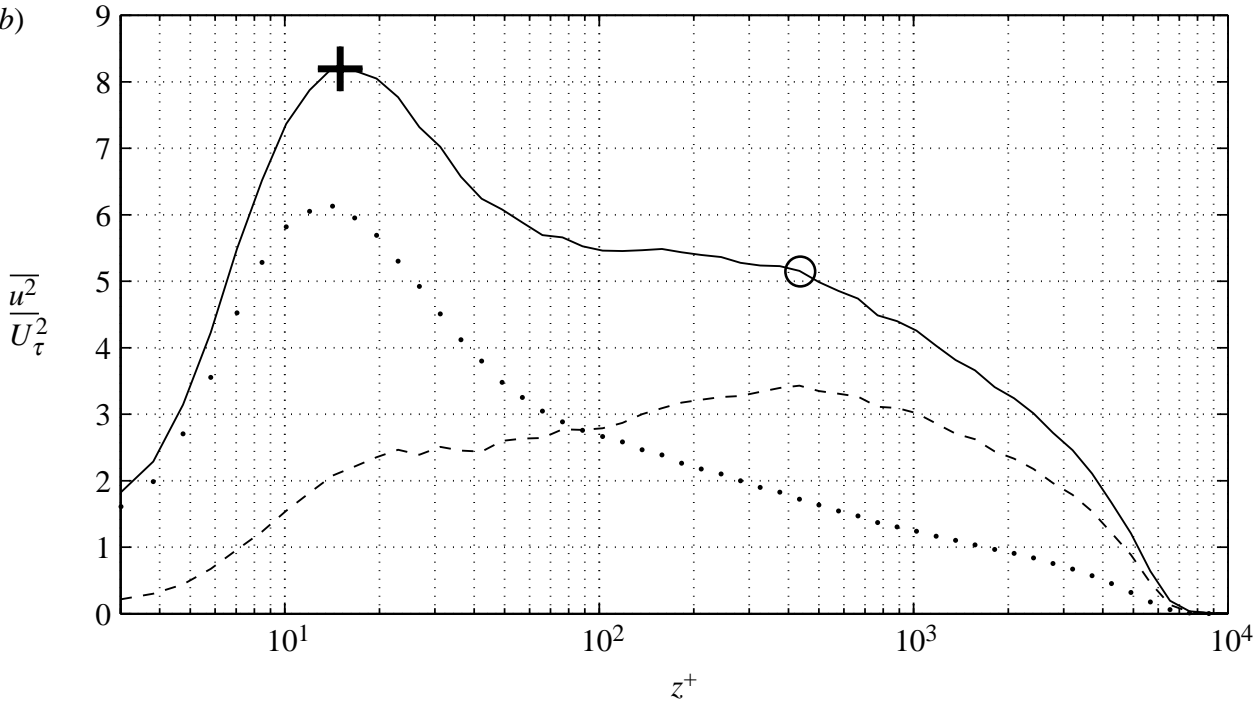

Figure 3. (a) Iso-contours of $k_{x} \phi_{u u} / U_{\tau}^{2}$ plotted on inner-scaled axes for (solid, filled) $R e_{\tau} \approx 1000$ and (symbols) $R e_{\tau} \approx 7300$. Contour levels are: small circle, 0.2; plus, 0.4; diamond, 0.6; triangle, 1.2 . Corresponding $R e_{\tau} \approx 1000$ contours are shown with solid lines. The large white '+' marks the inner peak $\left(\lambda_{x}^{+}=1000, z^{+}=15\right)$, and the white and black large open large circles mark the outer peak $\left(\lambda_{x}=6 \delta, z=0.06 \delta\right)$ for $R e_{\tau} \approx 1000$ and $R e_{\tau} \approx 7300$, respectively. (b) The corresponding streamwise turbulence intensity profiles at $R e_{\tau} \approx 7300$. Solid, broadband; dotted, small scales only, $\lambda_{x}^{+}<7300$; dashed, large scales only, $\lambda_{x}^{+}>7300$. The horizontal dashed line of plot $(a)$ shows the location of the spectral filter. 
high-speed event, and vice versa. Collectively, these very large features have been termed 'superstructures'. This highly elongated stripiness comprises relatively uniform low- and high-speed fluctuations of approximate magnitude $O\left( \pm 4 U_{\tau}\right)$ about the local mean. The meandering tendency of these features was used to explain why the (outer) energy peak in $k_{x} \phi_{u u}$ shows up at slightly lower wavelengths. These HWA rake experiments cover a Reynolds number range $1000 \leqq R e_{\tau} \lesssim 20000$. Using two-point correlations, it was shown that the size of the superstructure scales on boundary-layer thickness $\delta$. The spanwise rake approach has also been used to obtain similar measurements from the atmospheric surface layer (ASL). These experiments have confirmed that the same $\delta$-scaled log-region stripiness exists in the near-neutral ASL at $R e_{\tau} \approx 660000$ (see Hutchins \& Marusic in press, which includes an instantaneous example of the ASL superstructure that is almost $0.5 \mathrm{~km}$ in length). Ganapathisubramani et al. (2006) used side-by-side cameras and a coarse resolution $\left(i^{+} \approx 672\right.$, where $i$ is the interrogation window size) to image $8 \delta$ in the streamwise direction of a $R e_{\tau}=5600$ supersonic turbulent boundary layer. In doing so, they provide compelling PIV snapshots hinting at the true scale of the log-region structure. Again, they show very long meandering features (exceeding their $8 \delta$ field of view).

It is likely that this very large outer-scaled stripiness in the $u$ fluctuations (the superstructure) causes the outer peak in the pre-multiplied energy spectra $\left(k_{x} \phi_{u u}\right)$ as shown in figures 1 and 2 . While the spatial extent of these fluctuations scales very well with $\delta$ (hence the fixed location of the outer energy peak in $z / \delta$ and $\left.\lambda_{x} / \delta\right)$, an appropriate scaling for the magnitude of the fluctuations is not so clear. The increasing elevation with the Reynolds number of the outer peak in figures $1 b$ and $2 b$ indicates that friction velocity alone cannot correctly scale the streamwise energy of the superstructure, at least for the flow cases considered thus far. Regardless of the precise velocity scaling, the trend indicates that with the increasing Reynolds number, the energy due to the superstructure will become increasingly comparable to the energy due to the near-wall structure.

Up until fairly recently, the Reynolds number of DNS has been too low to clearly see the emergence of the very large scale outer structure. However, with ever increasing computer power, there has been a recent concerted drive towards numerical channel simulations at higher Reynolds numbers, and equally important, very large computational box sizes. Though one must exercise caution in comparing closed geometries with flat-plate boundary layers (see $\$ 2 a$ ), there is notable evidence of superstructures in these larger DNS. Through analysis of instantaneous DNS data at $R e_{\tau} \approx 640$, Abe et al. (2004) were able to conclude that very large structures exist in the outer layer, and that these structures are instantaneously visible in the surface shear-stress fluctuations. Iwamoto et al. (2005) also show instantaneous snapshots of very large scale structures interacting with the near-wall streamwise velocity field at $R e_{\tau}=2320$. Through simple filtering techniques applied to the DNS database of del Álamo et al. (2004), Hutchins \& Marusic (in press) present compelling evidence of a $20 \delta$ long log-region event, interacting with the near-wall region $\left(\right.$ at $\left.z^{+} \approx 15\right)$. From LES studies of pipe and channel flows, Tsubokura (2005) also noted that the logregion structure imposes outer-scaled energy onto the wall. It is well known that the inner-scaled peak in the streamwise turbulence intensity rises with the Reynolds number (DeGraaff \& Eaton 2000; Metzger \& Klewicki 2001; 
Metzger et al. 2001; Marusic \& Kunkel 2003). By low-pass filtering the $u$ signal at $z^{+}=15$ of a high Reynolds number boundary layer, Metzger \& Klewicki (2001) show that the additional energy in the near-wall streamwise fluctuations originates from low-wavenumber events. The DNS-observed instances of $(\delta$ scaled) superstructures interacting with (viscous-scaled) near-wall structures offer a clear instantaneous example of this phenomenon.

Figure $3 a$ shows iso-contours of pre-multiplied energy for the spectral surfaces shown previously in figures 1 and 2 . The shaded region is the energy surface at $R e_{\tau} \approx 1000$, with the four solid contours corresponding to $k_{x} \phi_{u u} / U_{\tau}^{2}=0.2,0.4,0.6$ and 1.2. The symbols show the corresponding contours for the $R e_{\tau} \approx 7300$ data. Since the contours are plotted on viscous-scaled axes $\left(z^{+}\right.$and $\left.\lambda_{x}^{+}\right)$, the location of the inner peak (shown by the plus sign) is fixed for both Reynolds numbers. For the rectangular region described by the limits $\lambda_{x}^{+} \lesssim 1000$ and $z^{+} \lesssim 100$, the two sets of contours show reasonable collapse on inner-scaled axes. However, if we look to higher values of $\lambda_{x}^{+}$, it becomes immediately obvious that for $z^{+}=15$ (the location of the near-wall peak in turbulent intensity), there is an increasing amount of low-wavenumber energy for the higher Reynolds number case. Furthermore, this energy is the footprint of the emergent outer energy site (as indicated by the emergent peak at $\lambda_{x} \approx 6 \delta, z \approx 0.06 \delta$ described by the $k_{x} \phi_{u u}=1.2 U_{\tau}^{2}$ contour). Using our simple bimodal model, the broadband turbulent intensity in the near-wall region is composed of a viscous-scaled component (the near-wall streaks) and a $\delta$-scaled component; figure $3 b$ clarifies this stance. Here, the fluctuating signals across the $R e_{\tau} \approx 7300$ boundary layer are decomposed into large- and small-scale fluctuations, using a spectral filter. In other words, Fourier coefficients greater than or less than $\lambda_{x}^{+}=7300\left(\lambda_{x}=\delta\right)$ are set to zero to obtain the respective small- and large-scale decomposed signals. The small-scale component (dotted line) accounts for the majority of the nearwall peak, after which it appears to decay in an approximately log-linear fashion throughout the logarithmic region. The large-scale energy, although contributing principally to the log region (peaking at $z \approx 0.06 \delta$, shown by the circle symbol), is strongly present in the near-wall region, accounting for approximately onequarter of the near-wall peak in streamwise intensity. This ratio is expected to increase with the Reynolds number, as the outer energy site grows (the strength of the superstructure fluctuations become increasingly comparable in magnitude to the near-wall structure).

\section{Amplitude modulation of near-wall region}

The decomposition for scales below and above $\lambda_{x}^{+} \approx 7300\left(\lambda_{x}=\delta\right)$ also reveal an interesting interaction between large- and small-scale motions in the boundary layer (we here define large and small scales as the component of the energy above or below the dashed line shown in figure $3 a$ ). It is noticed that the behaviour of the small-scale fluctuation is modulated by the large-scale component. Figure 4 illustrates this effect showing a typical fluctuating $u$ signal at $z^{+}=15$ decomposed into small and large components. Figure $4 a$ shows the original signal. It is noted that when the large-scale fluctuation (shown in figure $4 b$ ) is positive, the amplitude of the small scales (figure $4 c$ ) is larger; whereas when the large-scale fluctuation is negative, the small scales become increasingly quiescent. This effect 

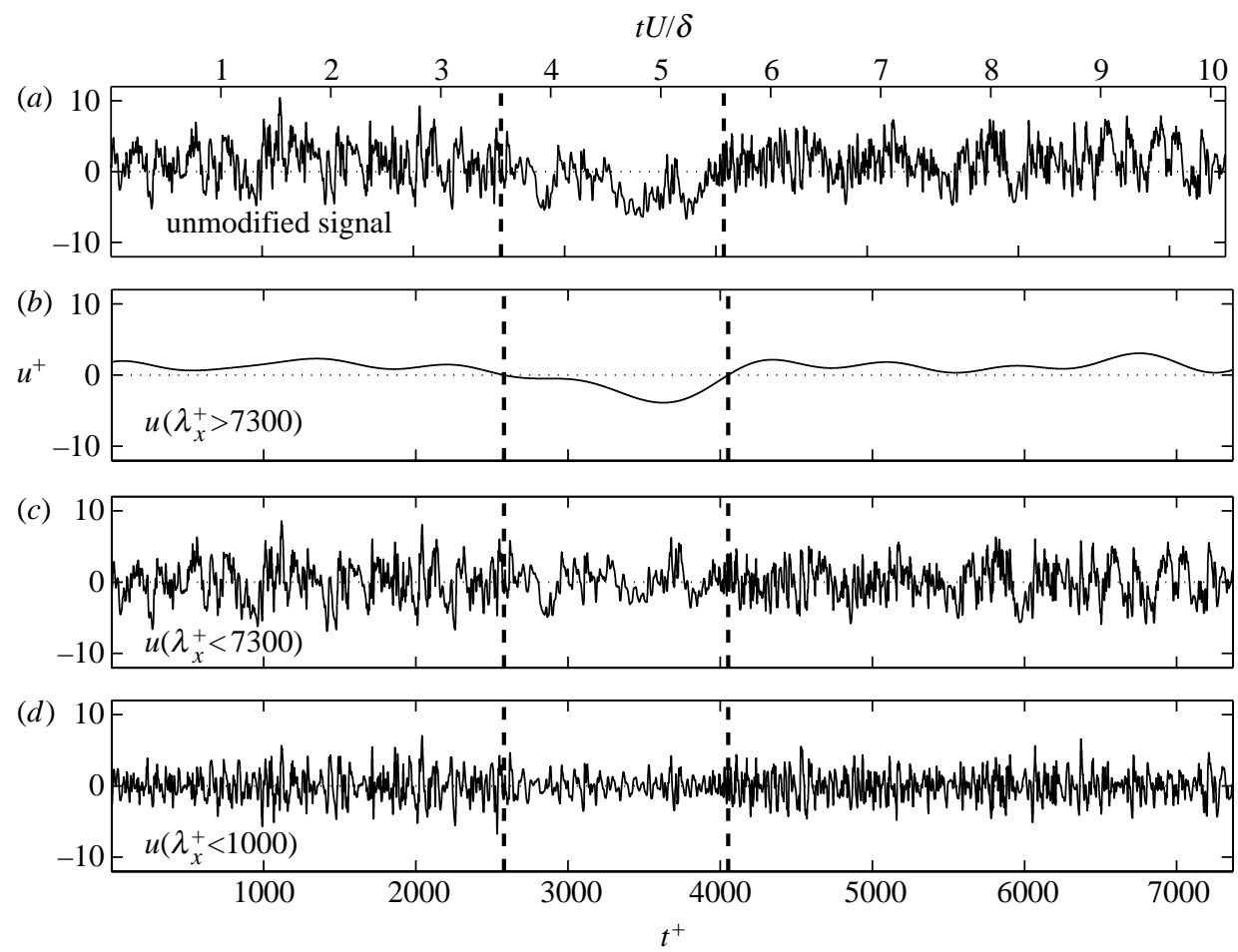

Figure 4. Example fluctuating $u$ signals in the near-wall region, $z^{+}=15\left(R e_{\tau} \approx 7300\right)$. (a) Raw fluctuating component, (b) large-scale fluctuation $\lambda_{x}^{+}>7300,(c)$ small-scale fluctuation $\lambda_{x}^{+}<7300$ and $(d)$ smaller-scale fluctuation $\lambda_{x}^{+}<1000$. Dashed vertical lines show region of negative largescale fluctuation.

is even clearer when a smaller cut-off filter is applied as in figure $4 d\left(\lambda_{x}^{+}<1000\right)$. Hutchins \& Marusic (in press) showed that the very low wavenumber motions at $z^{+}=15$ are strongly correlated with the log region. However, the data in figure 4 further indicate that the low-wavenumber motions (associated with the log-region structure) influence the near-wall $u$ fluctuations in a manner akin to pure amplitude modulation.

We can understand this by considering a simple sinusoidal carrier of frequency $f_{\mathrm{c}}$ modulated by a sinusoidal envelope of lower frequency $f_{\mathrm{e}}$. The energy in the Fourier transform of the modulated signal will appear at the original carrier frequency $f_{\mathrm{c}}$ and the two new frequencies $f_{\mathrm{c}}-f_{\mathrm{e}}$ and $f_{\mathrm{c}}+f_{\mathrm{e}}$. For this analogy, the large-scale component (figure $4 b$ ) can be considered the envelope, and the smallscales the carrier. Thus, even when the $u$ signal is low-pass filtered to remove the large-scale component, information relating to the large scales can remain as an envelope, applying an amplitude modulation of the small scales (as is discernable in figure $4 c, d)$. This highlights the need for caution when interpreting turbulence spectra. Though Fourier analysis is successful in sorting linearly superimposed signals, it will tend to mask modes that amplitude modulate, especially where the periods of the 'carrier' and 'envelope' are widely separated and have broadband energy. 
To determine the statistical significance of the low- and high-speed log region influences, we further decompose the small-scale signal into two sub-portions where the large-scale excursion is either positive or negative. Figure 5 shows the statistical contribution of these signals to the total small-scale intensity for all wall-normal stations across the boundary layer. Close to the wall, at the peak of production, the small scales are clearly more energetic under the influence of a positive large-scale fluctuation (and more quiescent under large negative fluctuation), which reaffirms the result from figure 4 . Indeed, at $z^{+}=15$, the fine-scale activity within large-scale accelerated regions is approximately $17 \%$ greater than within decelerated regions. Away from the wall (for $z^{+} \geq 300$ ), this trend reverses, and the greater proportion of small-scale activity occurs within the elongated low-speed portion of the superstructure. Although this reversal is subtle in figure 5, the result is nonetheless consistent and repeatable, proving insensitive to filter size. The precise location of the cross-over point is likely to be Reynolds number dependent.

Such behaviour is also apparent in DNS data at $R e_{\tau}=934$ (del Álamo et al. 2004), even though at these Reynolds numbers the footprint of the superstructure is relatively weak. Here, the same trends are evident in the $v$ and $w$ fluctuations, both of which are more active under positive large-scale excitation. Figure 6 shows simultaneous signals for all three velocity components along with the instantaneous Reynolds shear stress $u w$ at $z^{+}=15$. The small-scale activities for all signals are notably less intense during a large-scale negative $u$ fluctuation (between the vertical dashed lines). As an additional point, it is evident from figure 6 that the wall-normal velocity fluctuation (figure $6 b$ ) does not have a large-scale component, while the $u$ and $v$ components (figure $6 a, c$ ) do, as noted in energy spectra by Hoyas \& Jiménez (2006).

Therefore, in the near-wall region, we have observed that under large-scale high-speed events (footprint of the superstructure), the local instantaneous Reynolds stresses (all components: $u^{2}, v^{2}, w^{2}$ and $u w$ ) are amplified, while the opposite is true under large-scale low-speed events. This is as expected since the local shear rate near the wall (and hence input of vorticity from the wall) is higher under high-speed events as compared with low-speed superstructure signatures. The reversal in this behaviour away from the wall, as indicated in figure 5, is more difficult to explain. Above a certain point within the log region, a larger proportion of the small-scale energy is contained within the elongated lowspeed region of the superstructure (conversely, the high-speed region of the superstructure contains less). Ganapathisubramani et al. (2003) and Marusic \& Hutchins (2005) both show enhanced Reynolds shear-stress concentrations aligned within the elongated low-speed regions of the log layer. These concentrations are associated with vortex cores that appear to be clustered around the low-speed regions. In separate DNS studies, Tanahashi et al. (2004) and Kasagi et al. (2005) show complex clusters of fine-scale vortices existing within these elongated low-speed regions (at $R e_{\tau}=800$ and 1160, respectively), noting that in the log region, "the probability that coherent fine-scale eddies exist in low-speed streaks is higher than that in high-speed streaks'.

An interpretation of the small-scale behaviour shown in figures 4-6 is that the fine-scale eddies or compact swirling (or vortical) regions are distributed in some ordered way throughout the superstructure. Near the wall, there must be more 


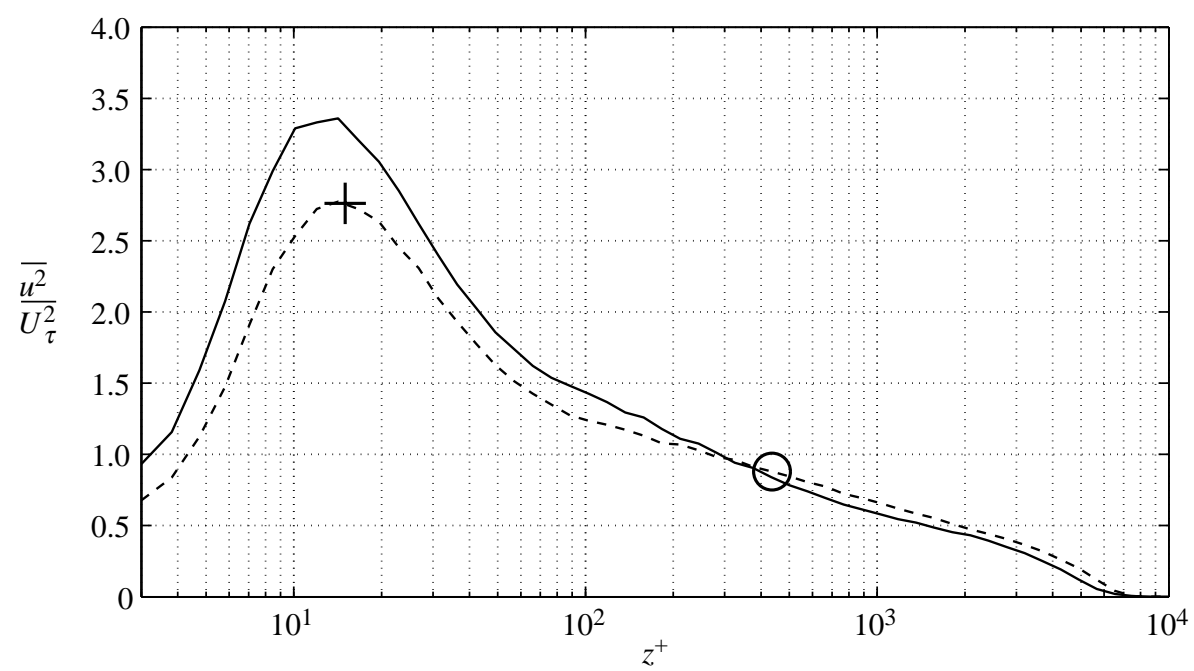

Figure 5. Turbulent intensity of decomposed small-scale energy under (dashed) negative and (solid) positive large-scale fluctuations. Curves sum to the dotted line on figure $3 b$.
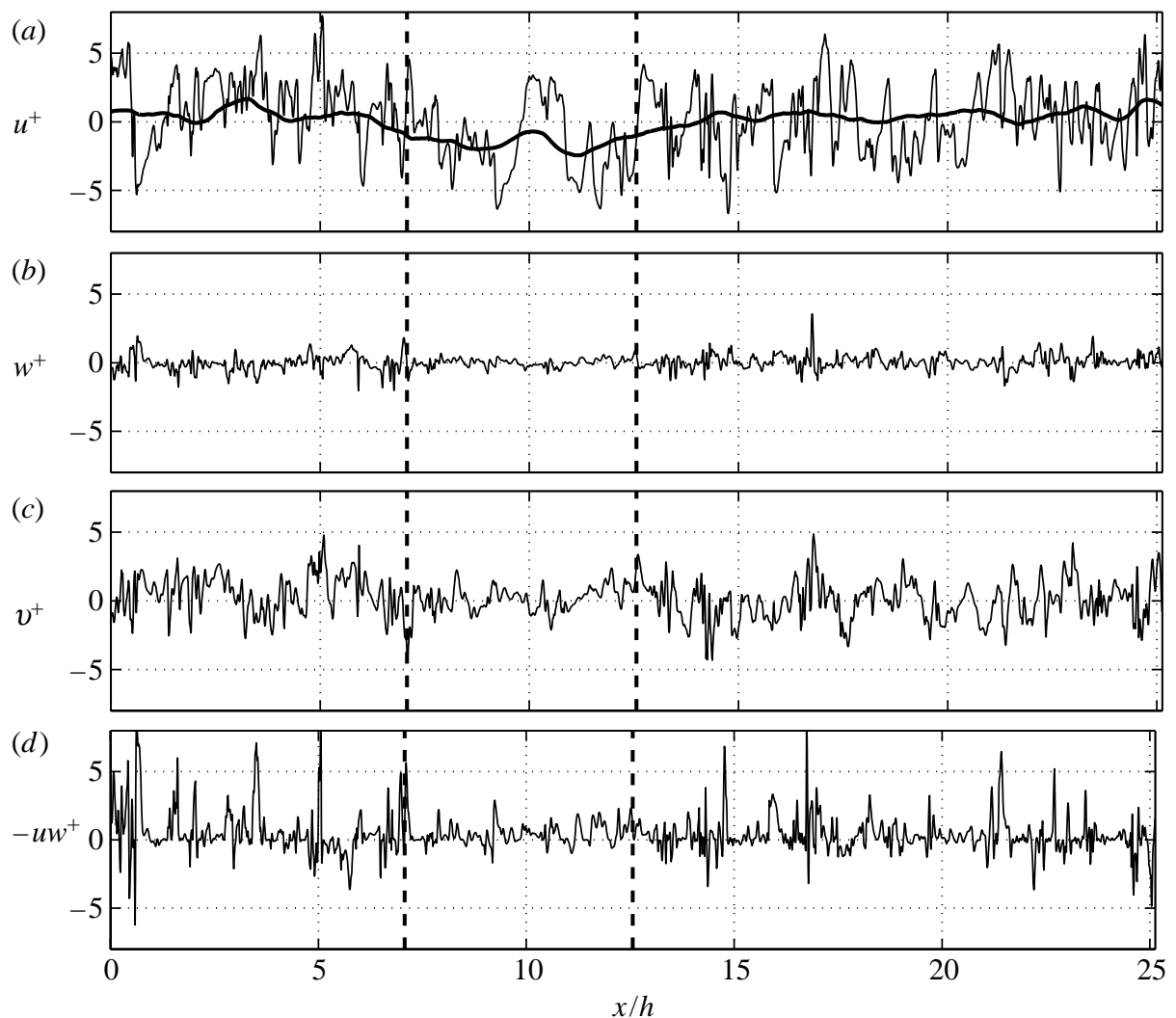

Figure 6. Example fluctuating velocity signals from the $R e_{\tau} \approx 950$ channel of del Álamo et al. (2004) at $z^{+}=15$. (a) Fluctuating $u$ component, thicker line shows large-scale component, $(b)$ wall-normal $w$ fluctuation, $(c)$ spanwise $v$ fluctuation, $(d)$ Reynolds shear-stress fluctuation. Dashed vertical lines show region of negative large-scale fluctuation. 

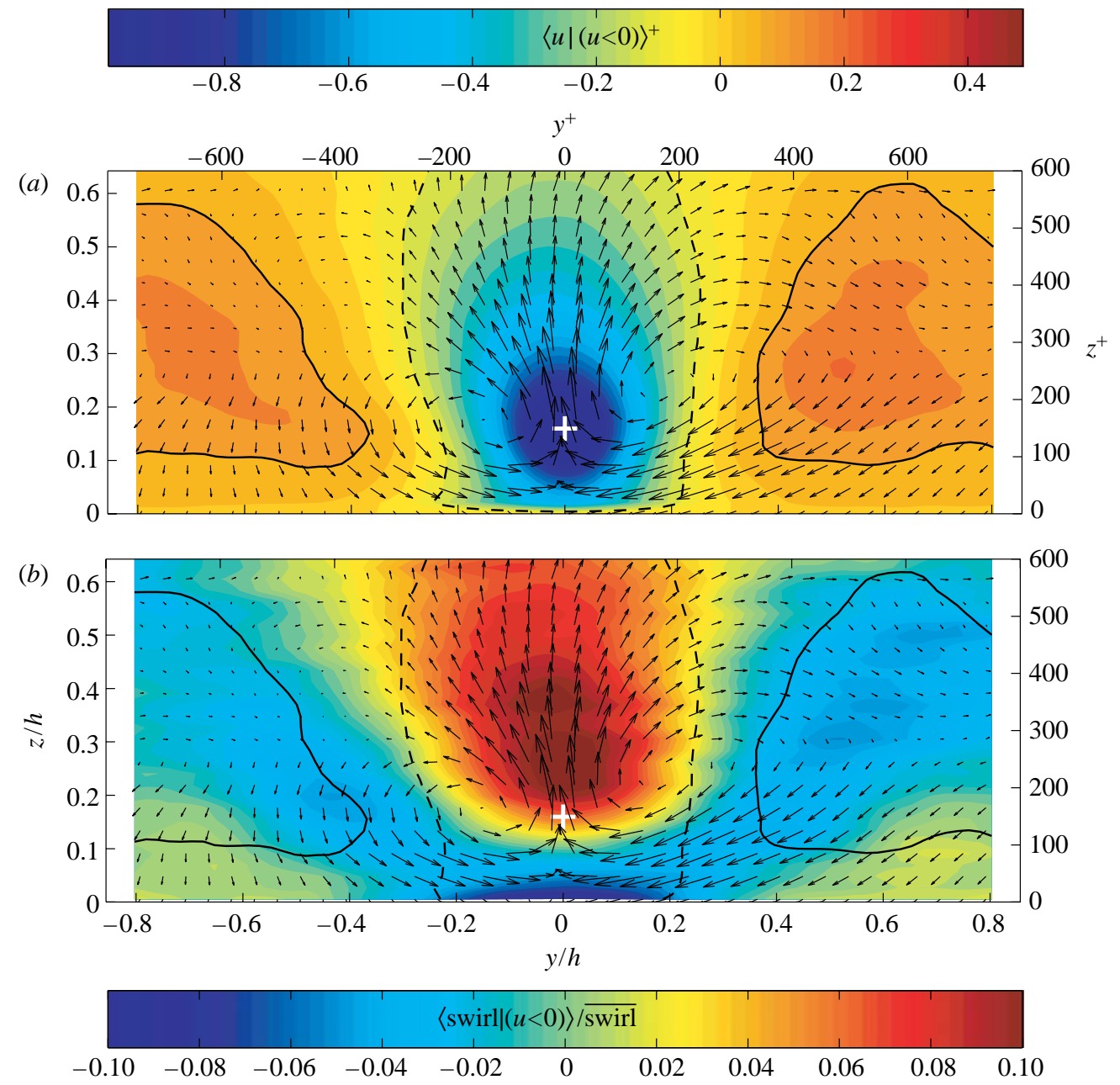

Figure 7. Conditionally averaged fields on the occurrence of a low-speed $u$ event at $z^{+} \approx 150$. (a) Conditional $u$ field $\langle u \mid(u<0)\rangle^{+}$. (b) Conditional swirl field $\langle\operatorname{swirl} \mid(u<0)\rangle^{+}$normalized by the mean swirl at each height. Contours show: solid, $u^{+}=0.1$; dashed, $u^{+}=-0.1$. Arrows show the vector due to $\langle v \mid(u<0)\rangle$ and $\langle w \mid(u<0)\rangle^{+}$.

swirling motions beneath the elongated high-speed region of the superstructure. And indeed for the DNS database, this is clear from an instantaneous comparison of swirl isosurfaces and local large-scale $u$ fluctuation. To test this statistically, a conditionally averaged view of the large-scale superstructure event has been calculated from the DNS channel flow database (del Álamo et al. 2004). Figure $7 a$ presents ensemble averaged contours of $u$ fluctuation conditioned on the occurrence of a large-scale negative $u$ fluctuation in the log layer $(u<0$ at $\left.z^{+}=150\right)$. A large low-speed region is flanked in $y$ by similar-sized high-speed events. This coherence extends for many channel heights in the streamwise direction (the form is similar to any standard two-point correlations from the log layer; e.g. Marusic \& Hutchins 2005). The superimposed vectors show the conditioned $v$ and $w$ components and indicate that the superstructure is associated with a large-scale counter-rotating roll mode. The dashed contour, 
drawn at $u^{+}=-0.1$, appears to be curtailed or 'splatted' onto the wall and is indicative of the wall footprint due to the superstructure (see Hutchins et al. 2005).

Figure $7 b$ shows colour contours of the ensemble-averaged swirl field conditioned on the same low-speed event (the ensemble is normalized by the mean swirl profile). Here, swirl is defined as the imaginary part of the complex eigenvector of the velocity gradient tensor and is shown to be a useful maker of compact swirling motions (Adrian et al. 2000a). Close to the wall in figure $7 b$, beneath the low-speed footprint of the superstructure, the ensembled swirl is reduced by over $10 \%$. On either side of this, under the high-speed events, there are regions of enhanced swirl. Away from the wall, this situation reverses and the low-speed region (shown by the dotted contour) is characterized by increased swirling activity throughout the upper log and wake layers. In contrast, the solid contours at this height, denoting the regions of positive $u$ fluctuation, encircle an area of reduced swirl. This conditionally averaged behaviour is consistent with the previous interpretation of the amplitude modulation shown in figures 4 and 5 , whereby the large-scale motions, originating in the log region, modulate the near-wall motions.

The amplitude modulation of small scales by the superstructure illustrates a complex nonlinear interaction across events that are distant in scale by several orders of magnitude. Such interaction is largely absent from a purely Fourier decomposed view of turbulence. There is little evidence of this amplitude modulation in the spectra of $w$ or $-\overline{u w}$, and so these components can appear to be disassociated from the large-scale events. On the contrary, figures 4-7 show that the generation and location of the small scales (and hence the fine-scale vortices) are very much connected with the largest scale structures in the boundary layer.

\section{Concluding remarks}

As we move to higher Reynolds numbers, the superstructure events are increasingly likely to dominate our view of turbulent coherent structures. The near-wall cycle, long regarded as autonomous, is actually flourishing (or languishing) under the influence of large-scale modulating events. The superstructures are not merely superimposed as a low-wavenumber mean shift onto the near-wall region. Rather, they appear to actively modulate the production of near-wall scales, and the extent to which it does so will increase with the Reynolds number (as the outer peak becomes increasingly comparable in energy to the inner peak). This has very obvious implications for those who seek to actively control turbulence (for drag reduction, mixing enhancement, etc.). In addition, these large scales also seem to play a crucial role in redistributing finescale turbulent motions throughout the boundary layer. At this stage, the mechanism for this redistribution is not clear, but the large-scale roll modes evident in figure 7 provide one possible explanation. The increasing influence of the superstructure with the Reynolds number is likely to challenge many of our assumptions concerning turbulent boundary layers, with small-scale turbulent fluctuations becoming increasingly inhomogeneous under the organizing influence of the largest scales. In addition, it is becoming clear that these large-scale 
structures will most probably play key roles in high Reynolds number boundarylayer interactions. Recently, Ganapathisubramani et al. (submitted) showed how such structures play a role in shock-boundary-layer interactions.

The data presented in this paper cover a significant range of Reynolds number and highlight the need for adequate scale separation when considering the coherent structure in wall turbulence. In future work, it would be worthwhile to consider how these findings relate to the asymptotic scaling issues as discussed elsewhere in this issue by McKeon \& Morrison (2007).

As a final comment, it is worthwhile mentioning how our findings of outer/inner region interaction relate to Townsend's (1976) theory of active and inactive motions, which are extensively referred to in this issue and in the wall-turbulence literature in general. We have purposely avoided using this terminology throughout the paper, since it tends to cause some confusion. Very often the term 'inactive' has been interpreted literally, or simply taken to mean negligible contribution to Reynolds shear stress. Here, we have clearly seen that the large-scale wall-parallel motions (in this case associated with increased streamwise velocity) do bring about an amplification or excitation of small-scale $u, v$ and $w$ fluctuations, in a manner that will produce increased (small-scale) Reynolds stress fluctuations beneath them. In addition to this, the superstructures that produce these motions also carry a significant fraction of the Reynolds shear stress in the logarithmic region. Thus, they are extremely active in a literal sense. However, the induced near-wall signatures of these structures are essentially of the 'inactive' type that Townsend refers to, since the induced velocity signature itself has a negligible wall-normal velocity component. Further discussion of inactive motions, related to the attached eddy hypothesis, is given in this issue by Nickels et al. (2007).

The authors wish to gratefully acknowledge the financial support of the National Science Foundation (CTS-0324898), the David and Lucile Packard Foundation and the Australian Research Council (DP-0663499).

\section{References}

Abe, H., Kawamura, H. \& Choi, H. 2004 Very large-scale structures and their effects on the wall shear-stress fluctuations in a turbulent channel flow up to $R e_{\tau}=640$. J. Fluids Eng. 126, 835-843. (doi:10.1115/1.1789528)

Adrian, R. J., Christensen, K. T. \& Lui, Z.-C. 2000 a Analysis and interpretation of instantaneous turbulent velocity fields. Exp. Fluids 29, 275-290. (doi:10.1007/s003489900087)

Adrian, R. J., Meinhart, C. D. \& Tomkins, C. D. $2000 b$ Vortex organization in the outer region of the turbulent boundary layer. J. Fluid Mech. 422, 1-54. (doi:10.1017/S0022112000001580)

del Álamo, J. C. \& Jiménez, J. 2003 Spectra of the very large anisotropic scales in turbulent channels. Phys. Fluids 15, 41-44. (doi:10.1063/1.1570830)

del Álamo, J. C., Jiménez, J., Zandonade, P. \& Moser, R. D. 2004 Scaling of the energy spectra of turbulent channels. J. Fluid Mech. 500, 135-144. (doi:10.1017/S002211200300733X)

Christensen, K. T. \& Adrian, R. J. 2001 Statistical evidence of hairpin vortex packets in wall turbulence. J. Fluid Mech. 431, 433-443. (doi:10.1017/S0022112001003512)

DeGraaff, D. B. \& Eaton, J. K. 2000 Reynolds number scaling of the flat-plate turbulent boundary layer. J. Fluid Mech. 422, 319-346. (doi:10.1017/S0022112000001713)

Ganapathisubramani, B., Longmire, E. K. \& Marusic, I. 2003 Characteristics of vortex packets in turbulent boundary layers. J. Fluid Mech. 478, 35-46. (doi:10.1017/S0022112002003270) 
Ganapathisubramani, B., Hutchins, N., Hambleton, W. T., Longmire, E. K. \& Marusic, I. 2005 Investigation of large-scale coherence in a turbulent boundary layer using two-point correlations. J. Fluid Mech. 524, 57-80. (doi:10.1017/S0022112004002277)

Ganapathisubramani, B., Clemens, N. T. \& Dolling, D. S. 2006 Large-scale motions in a supersonic boundary layer. J. Fluid Mech. 556, 271-282. (doi:10.1017/S0022112006009244)

Ganapathisubramani, B., Clemens, N. T. \& Dolling, D. S. Submitted. Effects of upstream boundary layer on the unsteadiness of shock induced separation.

Guala, M., Hommema, S. E. \& Adrian, R. J. 2006 Large-scale and very-large-scale motions in turbulent pipe flow. J. Fluid Mech. 554, 521-542. (doi:10.1017/S0022112006008871)

Hambleton, W. T., Hutchins, N. \& Marusic, I. 2006 Multiple plane PIV measurements in a turbulent boundary layer. J. Fluid Mech. 560, 53-64. (doi:10.1017/S0022112006000292)

Hoyas, S. \& Jiménez, J. 2006 Scaling of the velocity fluctuations in turbulent channels up to $R e_{\tau}=2003$. Phys. Fluids 18, 011702. (doi:10.1063/1.2162185)

Hutchins, N., Ganapathisubramani, B. \& Marusic, I. 2004 Dominant spanwise Fourier modes, and the existence of very large scale coherence in turbulent boundary layers. In Proc. 15th Australasian Fluid Mech. Conf., Sydney.

Hutchins, N., Hambleton, W. T. \& Marusic, I. 2005 Inclined cross-stream stereo particle image velocimetry measurements in turbulent boundary layers. J. Fluid Mech. 541, 21-54. (doi:10. $1017 /$ S0022112005005872)

Hutchins, N. \& Marusic, I. In press. Evidence of very long meandering features in the logarithmic region of turbulent boundary layers. J. Fluid Mech.

Iwamoto, K., Kasagi, N. \& Suzuki, Y. 2005 Direct numerical simulation of turbulent channel flow at $R e_{\tau}=2320$. In Proc. 6th Symp. Smart Control of Turbulence, Tokyo.

Jiménez, J. 1998 The largest scales of turbulent wall flows. In CTR Annual Research Briefs, pp. 943-945. Stanford University.

Kasagi, K., Fukagata, K. \& Suzuki, Y. 2005 Adaptive control of wall-turbulence for skin friction drag reduction and some consideration for high Reynolds number flows. In 2nd Int. Symp. Seawater Drag Reduction, Busan.

Kim, K. C. \& Adrian, R. 1999 Very large-scale motion in the outer layer. Phys. Fluids 11, 417-422. (doi:10.1063/1.869889)

Kline, S. J., Reynolds, W. C., Schraub, F. A. \& Rundstadler, P. W. 1967 The structure of turbulent boundary layers. J. Fluid Mech. 30, 741-773. (doi:10.1017/S0022112067001740)

Liu, Z., Adrian, R. J. \& Hanratty, T. J. 2001 Large-scale modes of turbulent channel flow: transport and structure. J. Fluid Mech. 448, 53-80.

Marusic, I. \& Hutchins, N. 2005 Experimental study of wall turbulence: implications for control. In Transition and turbulence control, vol. 8 (eds M. Gad-el-Hak \& H. M. Tsai) Lecture Notes Series, National University of Singapore. Singapore: World Scientific.

Marusic, I. \& Kunkel, G. J. 2003 Streamwise turbulence intensity formulation for flat-plate boundary layers. Phys. Fluids 15, 2461-2464. (doi:10.1063/1.1589014)

McKeon, B. J. \& Morrison, J. F. 2007 Asymptotic scaling in turbulent pipe flow. Phil. Trans. R. Soc. A 365, 771-787. (doi:10.1098/rsta.2006.1945)

Metzger, M. M. \& Klewicki, J. C. 2001 A comparative study of near-wall turbulence in high and low Reynolds number boundary layers. Phys. Fluids 13, 692. (doi:10.1063/1.1344894)

Metzger, M. M., Klewicki, J. C., Bradshaw, K. L. \& Sadr, R. 2001 Scaling the near-wall axial turbulent stress in the zero pressure gradient boundary layer. Phys. Fluids 13, 1819-1821. (doi:10.1063/1.1368852)

Nickels, T. B., Marusic, I., Hafez, S. \& Chong, M. S. 2005 Evidence of the $k_{1}^{-1}$ law in a highReynolds-number turbulent boundary layer. Phys. Rev. Lett. 95, 074 501. (doi:10.1103/ PhysRevLett.95.074501)

Nickels, T. B., Marusic, I., Hafez, S., Hutchins, N. \& Chong, M. S. 2007 Some predictions of the attached eddy model for a high Reynolds number boundary layer. Phil. Trans. R. Soc. A 365, 807-822. (doi:10.1098/rsta.2006.1950) 
Panton, R. L. 2001 Overview of the self-sustaining mechanisms of wall turbulence. Prog. Aerosp. Sci. 37, 341-383. (doi:10.1016/S0376-0421(01)00009-4)

Robinson, S. K. 1991 Coherent motions in turbulent boundary layers. Annu. Rev. Fluid Mech. 23, 601-639. (doi:10.1146/annurev.fl.23.010191.003125)

Spalart, P. R. 1988 Direct numerical simulation of a turbulent boundary layer upto $\operatorname{Re}_{\theta}=1410$. J. Fluid Mech. 187, 61-98. (doi:10.1017/S0022112088000345)

Tanahashi, M., Kang, S.-J., Miyamoto, T., Shiokawa, S. \& Miyauchi, T. 2004 Scaling law of fine scale eddies in turbulent channel flows up to $R e_{\tau}=800$. Int. J. Heat Fluid Flow 25, 331-340. (doi:10.1016/j.ijheatfluidflow.2004.02.016)

Tomkins, C. D. \& Adrian, R. J. 2003 Spanwise structure and scale growth in turbulent boundary layers. J. Fluid Mech. 490, 37-74. (doi:10.1017/S0022112003005251)

Tomkins, C. D. \& Adrian, R. J. 2005 Energetic spanwise modes in the logarithmic layer of a turbulent boundary layer. J. Fluid Mech. 545, 141-162. (doi:10.1017/S0022112005006397)

Townsend, A. A. 1976 The structure of turbulent shear flow. Cambridge, UK: Cambridge University Press.

Tsubokura, M. 2005 LES study on the large-scale motions of wall turbulence and their structural difference between plane channel and pipe flows. In Proc. Fourth Int. Symp. on Turbulence and Shear Flow Phenomena, TSFP4, Willamsburg, VA.

Zhou, J., Adrian, R. J., Balachandar, S. \& Kendall, T. M. 1999 Mechanisms for generating coherent packets of hairpin vortices in channel flow. J. Fluid Mech. 387, 353-396. (doi:10.1017/ S002211209900467X) 


\section{University Library}

\section{- M M I N E R VA A gateway to Melbourne's research publications}

Minerva Access is the Institutional Repository of The University of Melbourne

Author/s:

Hutchins, N;Marusic, I

Title:

Large-scale influences in near-wall turbulence

Date:

2007-03-15

Citation:

Hutchins, N. \& Marusic, I. (2007). Large-scale influences in near-wall turbulence.

PHILOSOPHICAL TRANSACTIONS OF THE ROYAL SOCIETY A-MATHEMATICAL PHYSICAL AND ENGINEERING SCIENCES, 365 (1852), pp.647-664. https://doi.org/10.1098/ rsta.2006.1942.

Publication Status:

Published

Persistent Link:

http://hdl.handle.net/11343/34784 